\title{
Size changes in miR-21 knockout mice: Geometric morphometrics on teeth, alveolar bone and mandible
}

\author{
UWE YACINE SCHWARZE ${ }^{1-4}$, YUXIN NI ${ }^{1,5}$, YANMIN ZHOU ${ }^{5}$, LUCIA TERLECKI-ZANIEWICZ ${ }^{6}$, \\ MARKUS SCHOSSERER ${ }^{4,6}$, MATTHIAS HACKL ${ }^{4,7}$, JOHANNES GRILLARI $^{4,6,8}$ and REINHARD GRUBER ${ }^{1,4,9}$ \\ ${ }^{1}$ Department of Oral Biology, School of Dentistry, Medical University of Vienna, A-1090 Vienna; \\ Departments of ${ }^{2}$ Orthopaedics and Trauma and ${ }^{3}$ Dental Medicine and Oral Health, Medical University of Graz, \\ A-8010 Graz; ${ }^{4}$ Austrian Cluster for Tissue Regeneration, A-1200 Vienna, Austria; ${ }^{5}$ Department of Stomatology, \\ Union Shenzhen Hospital, Huazhong University of Science and Technology, Shenzhen, Guangdong 518051, \\ P.R. China; ${ }^{6}$ Institute of Molecular Biotechnology, Department of Biotechnology, \\ University of Natural Resources and Life Sciences, A-1190 Vienna; ${ }^{7}$ TAmiRNA GmbH, \\ A-1110 Vienna; ${ }^{8}$ Ludwig Boltzmann Institute for Experimental and Clinical Traumatology, \\ A-1200 Vienna, Austria; ${ }^{9}$ Department of Periodontology, School of Dental Medicine, \\ University of Bern, 3010 Bern, Switzerland
}

Received August 18, 2020; Accepted October 27, 2020

DOI: $10.3892 / \mathrm{mmr} .2021 .11924$

\begin{abstract}
MicroRNA-21 (miR-21) is a small non-coding RNA that is differentially expressed during tooth development, particularly during amelogenesis. Although orthodontic tooth movement and the innate immune response are impaired, miR-21 knockout mice demonstrate no obvious skeletal phenotype. However, the consequence of miR-21 knockout on tooth phenotype and corresponding alveolar bone is unknown. The current study utilized landmark-based geometric morphometrics to identify anatomical dissimilarities of the three lower and upper molars, and the corresponding alveolar bone, in miR-21 knockout and wild-type control mice. The anatomical structures were visualized by microcomputer tomography. A total of 36 and 38 landmarks were placed on mandibular and maxillary molars, respectively. For the alveolar bone, 16 landmarks were selected on both anatomical sites. General Procrustes analysis revealed significantly smaller molars and dimensions of the alveolar bone in the mandible of the miR-21 knockout mice when compared with wild-type controls $(\mathrm{P}=0.03$ and $\mathrm{P}=0.04$, respectively). The overall dimension of the mandible was reduced by the lack of miR-21 $(\mathrm{P}=0.02)$. In the maxilla, the dimension of the alveolar bone was significant $(\mathrm{P}=0.02)$; however, this was not observed in the molars $(\mathrm{P}=0.36)$. Based on principal component analysis, no changes in shape for any
\end{abstract}

Correspondence to: Professor Reinhard Gruber, Department of Oral Biology, School of Dentistry, Medical University of Vienna, 2a Sensengasse, A-1090 Vienna, Austria

E-mail: reinhard.gruber@meduniwien.ac.at

Key words: microRNA-21, miR-21, tooth morphology, development, geometrics morphometrics of the anatomical sites were observed. Dental and skeletal jaw length were calculated and no prognathism was identified. However, the fluctuating asymmetry of the molars in the mandible and the maxilla was reduced in the miR-21 knockout mice by 38 and $27 \%$, respectively. Taken together, the results of the present study revealed that the molars in the mandible and the dimension of the respective alveolar bone were smaller in miR-21 mice compared with wild-type littermates, suggesting that miR-21 influences tooth development.

\section{Introduction}

MicroRNAs (miRNAs) are small non-coding RNAs which regulate gene expression at the post-transcriptional level in eukaryotes (1). miR-21 levels are comparatively high in mammalian cells and upregulated in association with human colon cancer and chronic lymphocytic leukemia cells $(2,3)$. This seems to be an extraordinary property of miR-21 since in a profiling of 540 clinical samples of cancer patients it was the only constantly upregulated miRNA (4). The sum of miR-21 characteristics may lead to its utilization as a diagnostic and prognostic biomarker for diverse types of cancer and as a potential therapeutic target (5). In result most studies on miR-21 focused on its association with cancer and clinical application. However, miR-21 expression also controls osteoblast-mediated bone formation and osteoclast-related bone remodeling. For instance, miR-21 can promote and reduce osteogenic differentiation in MC3T3-E1 cells (6) and human adipose mesenchymal cells (7), respectively. Alternatively, miR-21 was highly upregulated during osteoclast differentiation (8) and regulates RANKL-induced osteoclastogenesis (9). In addition, miR-21 was found at increased levels in sera and bone tissue of osteoporotic patients, and high levels of miR-21 support fracture healing in preclinical models (10). Mouse models have provided further insights into the biological function of miR-21 in vivo. 
Knockout mice of miR-21 showed delayed early healing of alveolar socket following tooth extraction (11), impaired bone regeneration of maxillary bone defects (12), and enhanced inflammatory osteolysis upon ligature-induced periodontitis (13). Orthodontic tooth movement is impaired in miR-21 knockout mice (14-16), likely because miR-21 deficiency inhibits osteoclast function (17). During tooth development, particularly during amelogenesis, miR-21-5p turned out to be differentially expressed (18). However, the consequences of miR-21 knockout on the tooth phenotype and the corresponding alveolar bone have not been investigated so far.

There is a large variation of the shape in molar crowns making the metameric evaluation difficult. Consequently geometric morphometrics (GMM), a multivariate statistical technique to provide a comprehensive description of morphology aspects based on landmarks (19) was introduced to the field $(20,21)$. GMM was originally used in anthropology $(22,23)$ but also applied in clinical research on orthodontic tooth movement (24). GMM is now also increasingly implemented in mouse genetics to define a phenotype. For example, the craniofacial shape of Twist $1^{+/}$mice and wild-type controls was analyzed by GMM. Twist $1^{+/-}$mice showed a consistent pattern of craniofacial dysmorphology affecting all major regions of the skull (25). GMM also revealed that Panx3 knockout mice have shorter diaphyseal shafts compared with wild-type littermates, and relatively larger areas of muscle attachment sites (24), overall supporting the use of GMM to identify anatomical changes of bones.

GMM has been used to investigate the tooth phenotype in mouse models. For example, phylogeny and adaptation affect the shape of molars of insular mice (26). Mouse dietary groups can be distinguished with the use of a GMM based on first upper molars (27). Likewise, the impact of p63 on tooth and jaw development was reported based on GMM (28). GMM was further applied to determine the impact of BMP7 on the shape of molars in mutant mice (29). Considering the involvement of miR-21 in tooth development and the alveolar bone to follow the anatomy of the teeth, we hypothesized that miR-21 affects the anatomy of the tooth and consequently also the dimensions of the alveolar bone.

\section{Materials and methods}

Animals and microcomputed tomography. miR-21 knockout mice were backcrossed six times into the C57BL/6J background and maintained by breeding heterozygous animals. Mice were fed standard chow and kept under controlled lighting conditions (12 h light, $12 \mathrm{~h}$ dark) at the Division of Biomedical Research at the Medical University of Vienna. Animal experiments were approved by the local animal welfare committee and the Austrian Federal Ministry for Science (GZ BMWFW-66.009/0080-WF/V/3b/2017). We had skulls of 5 female and 3 male littermates, each miR-21 knockout (KO) and corresponding wild-type (WT) controls. Mice were sacrificed at an age between 39 and 66 weeks with carbon dioxide (at a displacement of volume at 10-30\% / per min ensuring a standardized increase and a homogeneous distribution of the gas inside the modified cage in October 2015). After cessation of respiratory and cardiac movements (observation after $\geq 10 \mathrm{~min}$, at room air) decapitation was performed. The heads were fixed in $4 \%$ formaldehyde for $48 \mathrm{~h}$ and transferred into $70 \%$ ethanol. $\mu \mathrm{CT}$ with an isometric voxel size of $17.2 \mu \mathrm{m}$ was carried out using a $\mu$ CT50 device (Scanco Medical AG). The scanning of the skulls was done at $70 \mathrm{kVp} / 100 \mu \mathrm{As}$ with an integration time of $500 \mathrm{msec}$.

Data acquisition. Bone and tooth surfaces of these skulls were generated by using the half maximum height value (HMHV) as threshold in each individual. The gray values of the tissue of interest and the adjacent material are measured and the value half way between the highest and lowest value is used as threshold for the surface reconstruction. The HMHV give the value most close to reality and the best standardization for possible differences in density. We established six different landmark sets for the different regions, i) upper teeth, ii) lower teeth, iii) upper alveolar bone, iv) lower alveolar bone, v) the mandible as a whole and vi) landmarks for jaw lengths. A total of 36 and 38 landmarks were placed according to the anatomical structure on the occlusal surface and root furcation of mandibular and maxillary molars. There are also 8 landmarks on each upper and lower alveolar bone per side, 17 landmarks on each side of the mandible (Fig. 1). Surface reconstruction and landmark coordinate retrieval were performed using Amira (Version 6.1; Visage Imaging Inc). For the dental jaw distance, distance between the temporomandibular joint and the cusps of the first lower and upper molar were calculated. For the skeletal distance, distance between the temporomandibular joint and the lower alveolar inner point of the lower incisor and the prosthion were calculated for the skeletal distance.

Statistical analysis. We transferred the coordinates of the landmarks into a text file in morphologika format for analysis in the EVAN Toolbox 1.71 (www.evan-society.org). A generalized Procrustes analysis was performed to superimpose the landmark configurations, quantify centroid size, and calculate the Procrustes distances and Procrustes shape coordinates. Separate principal component analysis (PCA) with and without the natural log of centroid sizes provided a representation of morphometric variation across groups. We used the decision procedure of Bookstein, when $2 N \log (a / g)$ exceeds ' 2 ' the principal component is considered for interpretation below ' 2 ' the principle component is considered to only express noise $(30,31)$. Asymmetry which is described by Procrustes distance between any form and its reflected relabeling was used as an additional quantification of perturbed development via the formulas for total, directional, and fluctuating asymmetry (32). Normality tests and according to the outcome unpaired t-tests or Mann-Whitney tests were performed using GraphPad Prism version 8.0.0 for Windows, GraphPad Software, www.graphpad.com.

\section{Results}

Generalized Procrustes analysis of molars and the dimension of the alveolar bone reveals reduced sizes in $m i R-21$ knockout mice. To compare the size of molars a generalized Procrustes analysis was performed. Based on the 36 and 38 landmarks placed on mandibular and maxillary molars, 
A
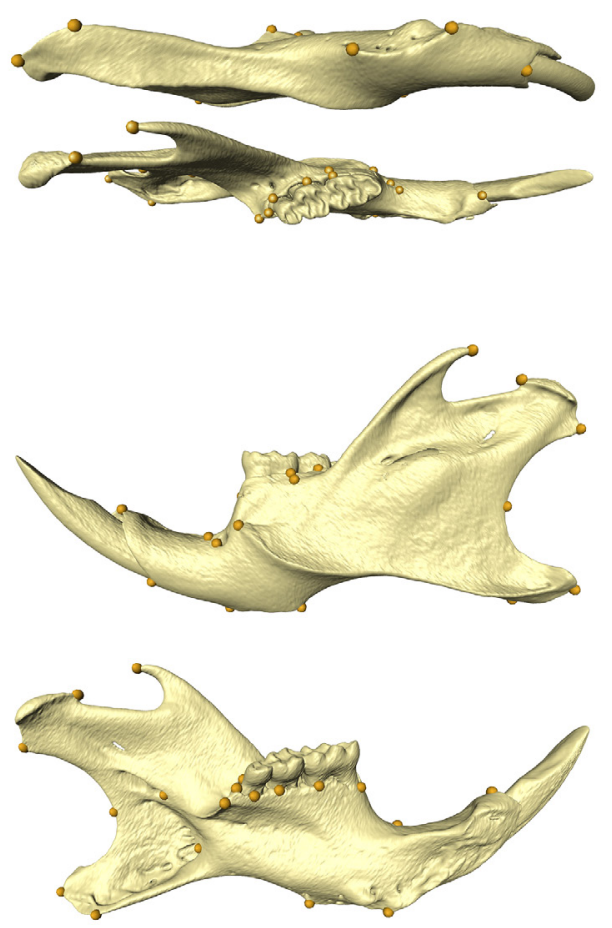

B

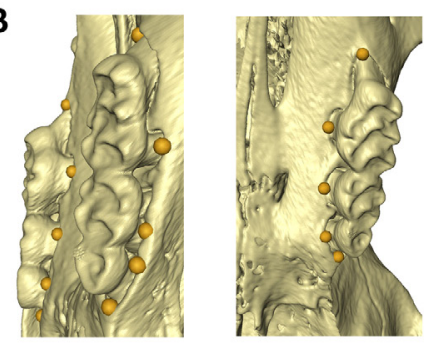

C

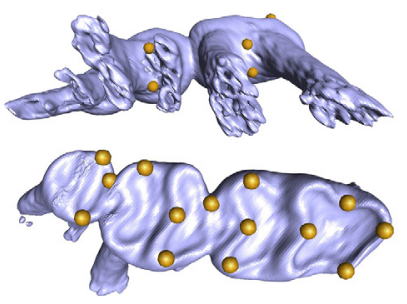

D

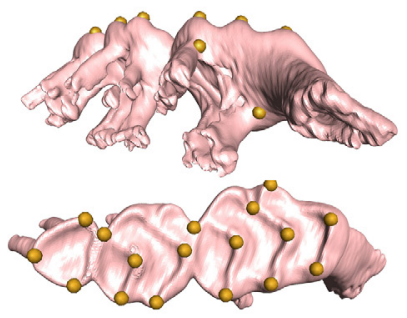

Figure 1. Landmark configuration (yellow dots) on mandible and teeth surface reconstruction. A total of 34 landmarks were placed according to anatomical structures on the (A) mandible, (B) 16 on each upper and lower alveolar bone, and a total of 36 and 38 on the occlusal surface and root furcation of (C) mandibular and (D) maxillary molars. Each region was analyzed separately.
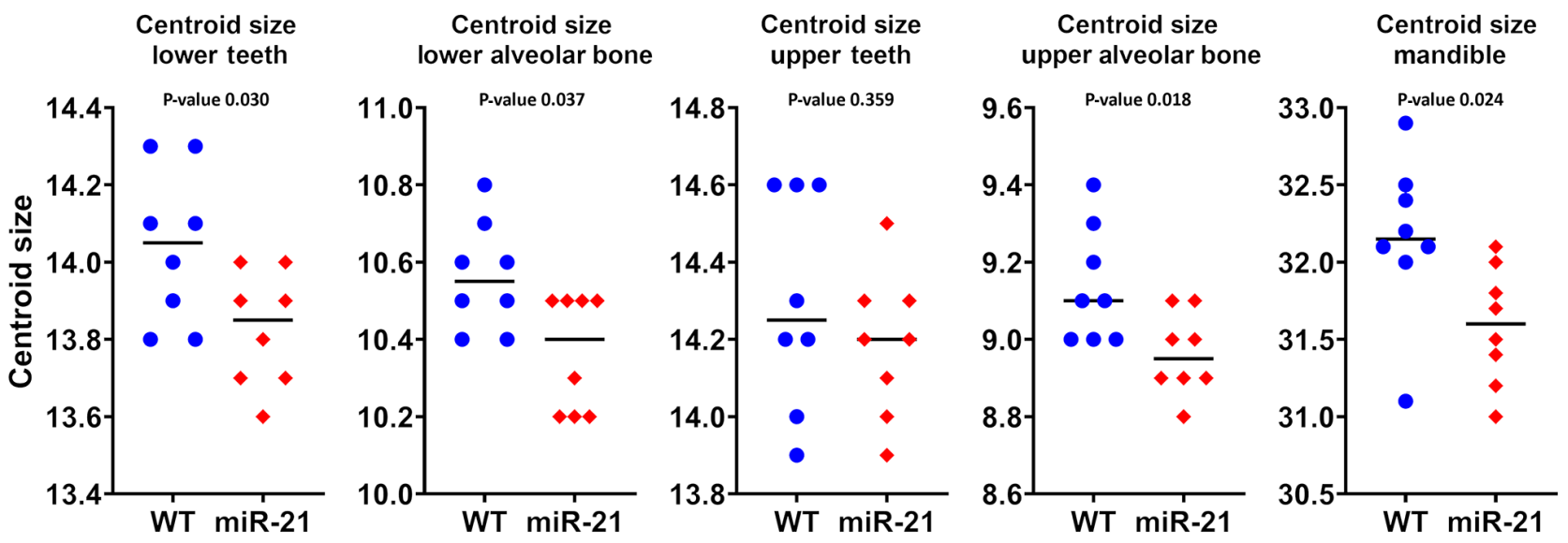

Figure 2. Centroid sizes of the investigated regions. Differences in centroid size reach a level of significance for the mandible, lower and upper alveolar bone and lower teeth but not for upper teeth $(\mathrm{n}=16)$. Black line indicated the median. WT, wild-type; miR-21, microRNA-21 knockout.

respectively, the molars in the mandible $(\mathrm{P}=0.02)$ but not on the maxilla $(\mathrm{P}=0.36)$ were significantly smaller in the miR-21 knockout mice compared to the wild-type controls. In line with these observations, the dimension of the alveolar bone of the mandible $(\mathrm{P}=0.03)$ and of the maxilla $(\mathrm{P}=0.02)$ was smaller in the miR-21 knockout mice when compared to the wild-type littermates. In addition, the dimension of the mandible was reduced as a consequence of the lack of miR-21 $(P=0.02)$. Fig. 2 shows the statistical values of centroid size. Taken together, the absence of miR-21 causes smaller molars and the respective alveolar bone in the mandible and in the maxilla.
Principal component analysis to assess changes in form and shape. We performed the PCA with (form space) and without (shape space) the natural log of centroid sizes, which integrates size and shape information to find a difference in the variance between miR-21 knockout mice and corresponding wild-type controls. The two groups were similar in form and shape at all anatomical sites investigated (lower molars, lower alveolar bone, upper molars, upper alveolar bone, and mandible). In Table I, the variance and noise criterion of principal component 1 and 2 are shown. The Procrustes distance values on the $\mathrm{PC} 1$ for the lower alveolar bone are significantly different with size $(\mathrm{P}=0.01)$ (Fig. 3). 
Table I. Variance in form and shape and noise criterion.

A, PC1

\begin{tabular}{|c|c|c|c|c|}
\hline Site & Variance in form $(\%)$ & $2 \mathrm{~N} \ln (\mathrm{a} / \mathrm{g})$ & Variance in shape $(\%)$ & $2 \mathrm{~N} \ln (\mathrm{a} / \mathrm{g})$ \\
\hline Lower molars & 34.3 & 1.8 & 25.9 & 1.3 \\
\hline Upper molars & 42.8 & $7.0^{\mathrm{a}}$ & 19.2 & 0.2 \\
\hline Lower alveo-lar bone & 35.8 & $3.5^{\mathrm{a}}$ & 23.1 & 0.7 \\
\hline Upper alveo-lar bone & 33.0 & $2.3^{\mathrm{a}}$ & 21.5 & 0.5 \\
\hline Mandible & 24.8 & 0.7 & 20.1 & 0.2 \\
\hline
\end{tabular}

B, PC2

\begin{tabular}{|c|c|c|c|c|}
\hline Site & Variance in form $(\%)$ & 2Nln (a/g) & Variance in shape $(\%)$ & $2 \mathrm{~N} \ln (\mathrm{a} / \mathrm{g})$ \\
\hline Lower molars & 17.3 & 1.3 & 14.4 & 0.2 \\
\hline Upper molars & 10.8 & 0.1 & 15.0 & 0.2 \\
\hline Lower alveo-lar bone & 13.8 & 0.3 & 15.4 & 0.1 \\
\hline Upper alveo-lar bone & 15.3 & 0.5 & 15.1 & 0.3 \\
\hline Mandible & 16.4 & 0.3 & 16.0 & 0.2 \\
\hline
\end{tabular}

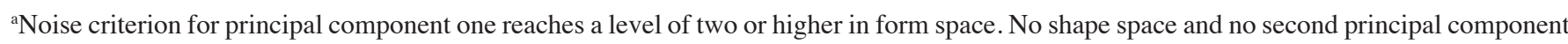
reached that limit and should not be used as explaining principal component. This indicates a high influence of size on the shape. Group separation on the principal component one of the regions with explaining value is presented in Fig. 3.

Lower alveolar bone

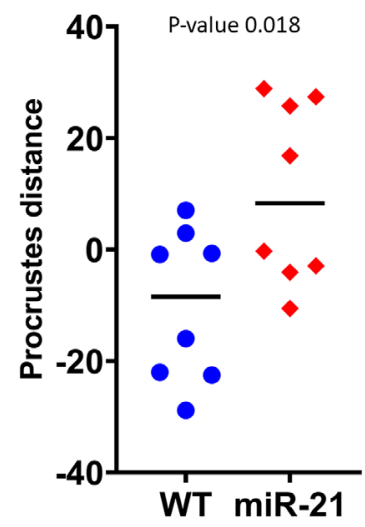

Upper teeth

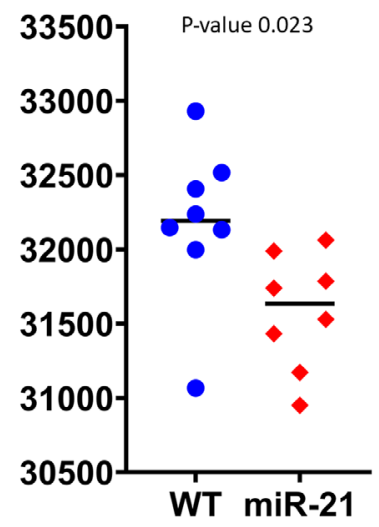

\section{Upper alveolar bone}

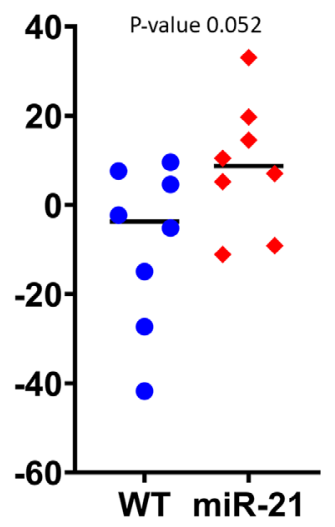

Figure 3. Distribution of Procrustes distance on the principal component 1 in form space. The differences of Procrustes distance on the PC1, including the natural logarithm of centroid size, was significant for lower and upper alveolar bone, but not for the upper teeth ( $\mathrm{n}=16$ ). Black line marks the median value. WT, wild-type; miR-21, microRNA-21 knockout.

Asymmetry analysis as a parameter for developmental stress and instability. The values presented are by their nature of calculation (32) group values, since directional and fluctuating asymmetry depend on the squared Procrustes distance between the original mean and the reflected mean of the landmarks. For that reason, significance values are not provided and the values should be interpreted as means. miR-21 knockout reduced the fluctuating asymmetry of the molars in the mandible and the maxilla by 38 and $27 \%$, respectively. Fluctuating asymmetry of the respective alveolar bone was also reduced in miR-21 knockout mice by 58 and $12 \%$ (Fig. 4)
Dental and skeletal jaw length as a parameter for genetic diseases. Jaw lengths of miR-21 knockout mice showed no differences in either dental or skeletal length when compared to the wild-type group (Fig. 5; $\mathrm{P}=0.72, \mathrm{P}=0.95$ ).

\section{Discussion}

Considering that microRNAs are involved in tooth development (33) and the notion that miR-21 is differentially expressed during this process, particularly during amelogenesis, it is reasonable to suggest that miR-21 knockout mice generate a tooth phenotype. However, miR-21 knockout mice 


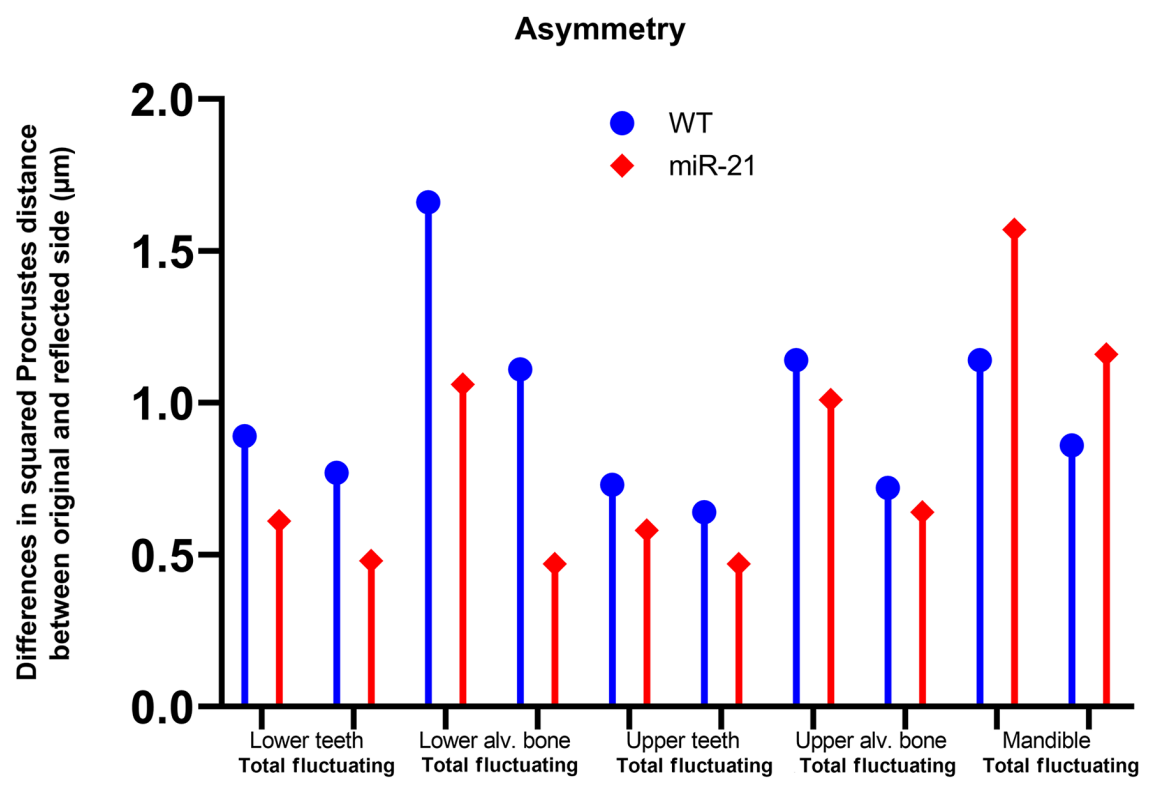

Figure 4. Total and fluctuating asymmetry of the five investigated regions. The group values for all regions demonstrated a reduced asymmetry in miR-21 knockout compared with wild-type mice. However, the mandible was the exception. WT, wild-type; miR-21, microRNA-21 knockout.
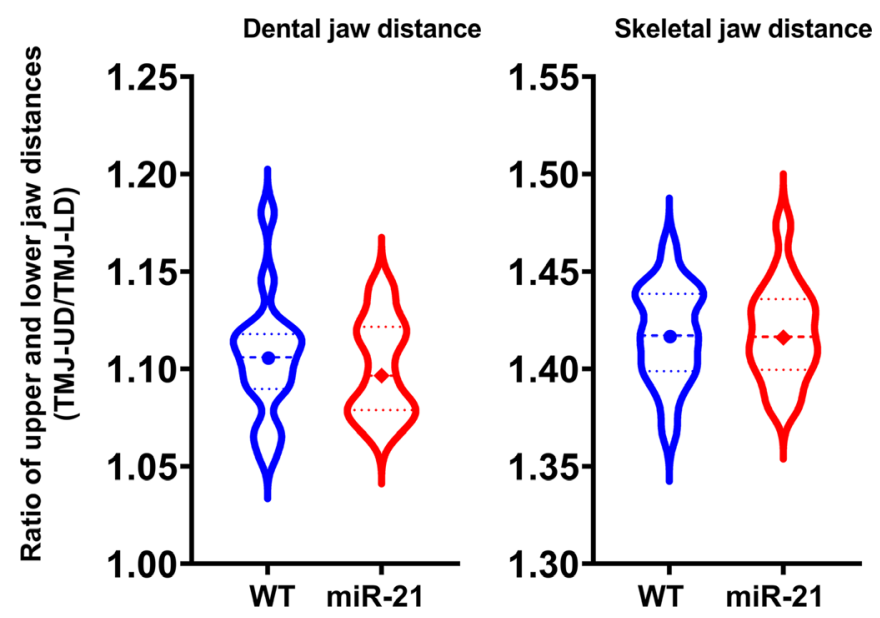

Figure 5. Dental and skeletal jaw distances. For dental jaw distance, the distance between the temporomandibular joint and the cusps of the first lower and upper molar were calculated. For skeletal distance, the distance between the temporomandibular joint and the lower alveolar inner point of the lower incisor and the prosthion were calculated. The ratio of these distances is presented. There was no difference between jaw distance ratios in WT and miR-21 mice. The broken line indicates the median, dotted lines indicate quartiles. WT, wild-type; miR-21, microRNA-21 knockout; TMJ, temporomandibular joint; UD, upper jaw distance; LD, lower jaw distance.

show a normal skeletal phenotype suggesting that, if at all, only marginal changes in tooth morphology can be expected. Bearing in mind that metameric evaluation of teeth is problematic, it requires GMM to reveal if miR-21 plays indeed a role during tooth development. According to this landmark-based statistical method, we demonstrate that the molars and the alveolar bone in the mandible and in the maxilla are smaller in the miR-21 knockout mice when compared to the wild-type controls. No changes in shape were noticed. miR-21 knockout also reduced the fluctuating asymmetry of the molars in both, the mandible and the maxilla suggesting a moderate effect on tooth development. Dental and skeletal jaw length can be influenced by genetic diseases (34), however, dental and skeletal jaw length showed no difference between the groups. Taken together, our data suggest that miR-21 affects, even though at a moderate level, molar development and the dimensions of the corresponding alveolar bone in mice.

Our findings basically support the ground tenor that microRNAs regulate tooth morphogenesis by fine-tuning the signaling network (33). Our research is among the pioneer study using GMM to identify the impact of miRNAs on tooth and alveolar bone morphology. The present study extends the use of GMM in mouse dental research such as phylogeny and shape adaptation of molars of insular mice and to distinguish dietary groups based on first upper molars. We applied GMM not only on molars but also on the respective alveolar bone in mouse models. This research is consistent with previous work with GMM to detect size shape and fluctuating asymmetry of the mandible and teeth in mouse models $(35,36)$. Fluctuating asymmetry is considered to be the product of developmental stress and instability, caused by both genetic and environmental stressors (37). A gene defect which results in the absence of a protein may cause developmental stress or uncanalized development leading to the inability of an organism to compensate (canalize) this stress which is often associated with excess morphological variance or higher asymmetry $(37,38)$. miR-21 knockout mice present here with less asymmetry which is the opposite. One reason to explain this could be that miR21 does not code for a protein but as a micro RNA is a regulator by itself. The interaction with other regulating mechanism could result in an imbalance of tissue apposition and therefore higher asymmetry in the wild-type. A second reason could be the smaller size of the more symmetrical tissue. At the level of hard tissue, failure to produce perfect symmetry is manifested by different apposition rates, different tooth eruption and suture fusion times in the developing organism. With less tissue mass, there seems to be a lower probability of failure in the symmetric apposition of tissue. The opposite was found 
in Sost KO mice where more bone mass lead to higher asymmetry (34).

A study limitation is that the presented work remains descriptive; thus, we have no explanation about the underlying molecular and cellular mechanisms that cause the smaller size of teeth in miR-21 knockout mice. However, new hypotheses originate from our cross search of genes regulated by miR-21 by miRWalk 2.0 and genes associated with GO terms by amigo.geneontology.org (39) for 'tooth': Four target genes were identified, namely peroxisome proliferator-activated receptor alpha (PPARA), endoribonuclease dicer (DICER1), activating transcription factor 2 (ATF2), and osteoprotegerin (TNFRSF11B, OPG). Interestingly, and similar to the dental phenotype of miR-21 knockout mice, a slight decrease in the size of the molars was observed in PPARA knockout mice. Thus, miR-21 might exert its function during tooth development by modulation of PPARA translational activity. DICER1 is involved in the biogenesis of most small RNAs, including miR-21 and plays a central role in tooth development. ATF2 activation occurs in the late secretion phase of ameloblasts apical to the transition zone of rat incisors OPG production by the dental follicle likely affects the alveolar bone resorption needed for tooth eruption. The present observations are a primer for a more detailed analysis on the expression changes of the putative target genes in miR-21 mice, with a particular focus on the cell involved in tooth formation (40).

Matrix metalloproteinases (MMPs) might offer another link between miR-21 and tooth anatomy. Mice deficient for MMP14, the membrane-type 1 metalloproteinase (MT1-MMP), have impaired tooth eruption and root elongation (41), and the expression of MT1-MMP was enhanced by miR-21 mimics in mesenchymal cells (42). Also, MMP20 and kallikrein-related peptidase 4 (KLK4) are required to harden enamel (43). There are potential miRNA-binding sites in the 3'-untranslated region of several MMPs (44) and miRNAs can participate in MMP regulation at the posttranscriptional level and change the expression of MMP genes (45). miR-21 promotes upregulation of MMP2 and MMP9 in human hepatocellular and pancreatic carcinoma cells $(46,47)$. It would be worth studying a possible involved of miR-21 in the regulation of MMP20 and KLK4. In general, the association of genes that play a role in tooth development being modulated by miR-21 might be the basis for future research.

The results presented here demonstrate that the molars and the respective alveolar bone in the mandible are significantly smaller in the miR-21 knockout mice compared to the wild-type controls. Shape changes were not found but a reduced asymmetry for either of the anatomical sites. It will now be critical to determine the molecular and cellular mechanisms how miR-21 affects tooth growth.

\section{Acknowledgements}

The authors would like to thank Ms. Astrid Fabry, (Center of Biomedical Research, Department for Laboratory Animal Science and Genetics, Medical University of Vienna, Austria) for excellent assistance with animal care. The authors would also like to acknowledge Professor Eric N. Olson (UT Southwestern, Dallas, TX, USA) for providing miR-21 knockout animals.

\section{Funding}

Yuxin Ni received a Scholarship from the Eurasia-Pacific Uninet. The present study was supported by the Osteology Foundation (grant no. YG 15-244), the Austrian Science Fund (grant no. 4072-B28) and Herzfelder'sche Familienstiftung (grant nos. P30623 and I2514-B28).

\section{Availability of data and materials}

The datasets used and/or analyzed during the current study are available from the corresponding author on reasonable request.

\section{Authors' contributions}

UYS, YN, JG and RG made substantial contributions to conception and design. UYS, YN, YZ, LTZ, MS, MH and JG acquired the landmark data. UYS, YN and RG analyzed and interpreted the data. UYS, YN and RG drafted the manuscript. UYS, YN, YZ, LTZ, MS, MH, JG and RG critically revised the manuscript for important intellectual content. UYS, YN, YZ, LTZ, MS, MH, JG and RG gave final approval of the version to be published. All authors read and approved the final manuscript.

\section{Ethics approval and consent to participate}

Animal experiments were approved by the local animal welfare committee and the Austrian Federal Ministry for Science (approval no. GZ BMWFW-66.009/0080-WF/V/3b/2017).

\section{Patient consent for publication}

Not applicable.

\section{Competing interests}

JG and MH are co-founders of TAmiRNA GmbH. The remaining authors declare that they have no competing interests.

\section{References}

1. Griffiths-Jones S, Grocock RJ, van Dongen S, Bateman A and Enright AJ: miRBase: microRNA sequences, targets and gene nomenclature. Nucleic Acids Res 34: D140-D144, 2006.

2. Feng YH, Wu CL, Tsao CJ, Chang JG, Lu PJ, Yeh KT, Uen YH, Lee JC and Shiau AL: Deregulated expression of sprouty2 and microRNA-21 in human colon cancer: Correlation with the clinical stage of the disease. Cancer Biol Ther 11: 111-121, 2011.

3. Fulci V, Chiaretti S, Goldoni M, Azzalin G, Carucci N, Tavolaro S, Castellano L, Magrelli A, Citarella F, Messina M, et al: Quantitative technologies establish a novel microRNA profile of chronic lymphocytic leukemia. Blood 109: 4944-4951, 2007.

4. Volinia S, Calin GA, Liu CG, Ambs S, Cimmino A, Petrocca F, Visone R, Iorio M, Roldo C, Ferracin M, et al: A microRNA expression signature of human solid tumors defines cancer gene targets. Proc Natl Acad Sci USA 103: 2257-2261, 2006.

5. Feng YH and Tsao CJ: Emerging role of microRNA-21 in cancer. Biomed Rep 5: 395-402, 2016.

6. Li H, Yang F, Wang Z, Fu Q and Liang A: MicroRNA-21 promotes osteogenic differentiation by targeting small mothers against decapentaplegic 7. Mol Med Rep 12: 1561-1567, 2015. 
7. Weilner S, Skalicky S, Salzer B, Keider V, Wagner M, Hildner F, Gabriel C, Dovjak P, Pietschmann P, Grillari-Voglauer R, et al: Differentially circulating miRNAs after recent osteoporotic fractures can influence osteogenic differentiation. Bone 79 43-51, 2015.

8. Kagiya T and Nakamura S: Expression profiling of microRNAs in RAW264.7 cells treated with a combination of tumor necrosis factor alpha and RANKL during osteoclast differentiation. J Periodontal Res 48: 373-385, 2013

9. Sugatani T, Vacher J and Hruska KA: A microRNA expression signature of osteoclastogenesis. Blood 117: 3648-3657, 2011.

10. Sun Y, Xu L, Huang S, Hou Y, Liu Y, Chan KM, Pan XH and Li G: mir-21 overexpressing mesenchymal stem cells accelerate fracture healing in a rat closed femur fracture model. BioMed Res Int 2015: 412327, 2015

11. Strauss FJ, Stähli A, Kobatake R, Tangl S, Heimel P, Apaza Alccayhuaman KA, Schosserer M, Hackl M, Grillari J and Gruber R: miRNA-21 deficiency impairs alveolar socket healing in mice. J Periodontol: May 12, 2020 (Epub ahead of print). doi: 10.1002/JPER.19-0567.

12. Wang $\mathrm{H}$, Wang $\mathrm{H}$, Li X, Zhang Z, Zhao X, Wang $\mathrm{C}$ and Wei F: MicroRNA-21 promotes bone reconstruction in maxillary bone defects. J Oral Rehabil: Sep 25, 2019 (Epub ahead of print). doi: 10.1111/joor.12896 2019.

13. Zhou W, Su L, Duan X, Chen X, Hays A, Upadhyayula S, Shivde J, Wang H, Li Y, Huang D, et al: MicroRNA-21 down-regulates inflammation and inhibits periodontitis. Mol Immunol 101: 608-614, 2018

14. Wu L, Su Y, Lin F, Zhu S, Wang J, Hou Y, Du J, Liu Y and Guo L: MicroRNA-21 promotes orthodontic tooth movement by modulating the RANKL/OPG balance in T cells. Oral Dis 26 : 370-380, 2020

15. Chen N, Sui BD, Hu CH, Cao J, Zheng CX, Hou R, Yang ZK, Zhao P, Chen Q, Yang QJ, et al: MicroRNA-21 Contributes to Orthodontic Tooth Movement. J Dent Res 95: 1425-1433, 2016.

16. Barnett RE, Conklin DJ, Ryan L, Keskey RC, Ramjee V, Sepulveda EA, Srivastava S, Bhatnagar A and Cheadle WG: Anti-inflammatory effects of miR-21 in the macrophage response to peritonitis. J Leukoc Biol 99: 361-371, 2016

17. Hu CH, Sui BD, Du FY, Shuai Y, Zheng CX, Zhao P, Yu XR and Jin Y: miR-21 deficiency inhibits osteoclast function and prevents bone loss in mice. Sci Rep 7: 43191, 2017.

18. Yin K, Hacia JG, Zhong Z and Paine ML: Genome-wide analysis of miRNA and mRNA transcriptomes during amelogenesis. BMC Genomics 15: 998, 2014

19. Klingenberg CP: Size, shape, and form: Concepts of allometry in geometric morphometrics. Dev Genes Evol 226: 113-137, 2016.

20. Gómez-Robles A, Martinón-Torres M, Bermúdez de Castro JM, Margvelashvili A, Bastir M, Arsuaga JL, Pérez-Pérez A, Estebaranz F and Martínez LM: A geometric morphometric analysis of hominin upper first molar shape. J Hum Evol 53 272-285, 2007

21. Gunz P and Mitteroecker P: Semilandmarks: A method for quantifying curves and surfaces. Hystrix 24: 103-109, 2013.

22. Gómez-Robles A, Martinón-Torres M, Bermúdez de Castro JM, Prado L, Sarmiento S and Arsuaga JL: Geometric morphometric analysis of the crown morphology of the lower first premolar of hominins, with special attention to Pleistocene Homo. J Hum Evol 55: 627-638, 2008

23. Badawi-Fayad J and Cabanis EA: Three-dimensional Procrustes analysis of modern human craniofacial form. Anat Rec (Hoboken) 290: 268-276, 2007.

24. Caskenette D, Penuela S, Lee V, Barr K, Beier F, Laird DW and Willmore KE: Global deletion of Panx3 produces multiple phenotypic effects in mouse humeri and femora. J Anat 228: 746-756, 2016.

25. Parsons TE, Weinberg SM, Khaksarfard K, Howie RN, Elsalanty M, Yu JC and Cray JJ Jr: Craniofacial shape variation in Twist1 ${ }^{+/-}$mutant mice. Anat Rec (Hoboken) 297: 826-833, 2014

26. Ledevin R, Chevret P, Ganem G, Britton-Davidian J, Hardouin EA, Chapuis JL, Pisanu B, da Luz Mathias M, Schlager S, Auffray JC, et al: Phylogeny and adaptation shape the teeth of insular mice. Proc Biol Sci 283: 283, 2016.

27. Gómez Cano AR,HernándezFernández Mand Alvarez-SierraMA Dietary ecology of Murinae (Muridae, Rodentia): A geometric morphometric approach. PLoS One 8: e79080, 2013.
28. Paradis MR, Raj MT and Boughner JC: Jaw growth in the absence of teeth: The developmental morphology of edentulous mandibles using the p63 mouse mutant. Evol Dev 15: 268-279, 2013.

29. Zurowski C, Jamniczky H, Graf D and Theodor J: Deletion/loss of bone morphogenetic protein 7 changes tooth morphology and function in Mus musculus: Implications for dental evolution in mammals. R Soc Open Sci 5: 170761, 2018.

30. Bookstein FL: Measuring and Reasoning: Numerical Inference in the Sciences. University Press, Cambridge, 2014.

31. Coquerelle M, Bookstein FL, Braga J, Halazonetis DJ, Weber GW and Mitteroecker P: Sexual dimorphism of the human mandible and its association with dental development. Am J Phys Anthropol 145: 192-202, 2011.

32. Mardia kV, Bookstein FL and Moreton IJ: Statistical assessment of bilateral symmetry of shapes. Biometrika 87: 285-300, 2000.

33. Sehic A, Tulek A, Khuu C, Nirvani M, Sand LP and Utheim TP: Regulatory roles of microRNAs in human dental tissues. Gene 596: 9-18, 2017.

34. Schwarze UY, Dobsak T, Gruber R and Bookstein FL: Anatomical similarity between the Sost-knockout mouse and sclerosteosis in humans. Anat Rec 303: 2295-2308, 2020.

35. Keller JM, Allen DE, Davis CR and Leamy LJ: 2,3,7,8-Tetrachlorodibenzo-p-dioxin affects fluctuating asymmetry of molar shape in mice, and an epistatic interaction of two genes for molar size. Heredity 98: 259-267, 2007.

36. Klingenberg CP, Leamy LJ, Routman EJ and Cheverud JM: Genetic architecture of mandible shape in mice: Effects of quantitative trait loci analyzed by geometric morphometrics. Genetics 157: 785-802, 2001.

37. Schaefer K, Lauc T, Mitteroecker P, Gunz P and Bookstein FL: Dental arch asymmetry in an isolated Adriatic community. Am J Phys Anthropol 129: 132-142, 2006.

38. Wilkins AS: Canalization: A molecular genetic perspective. BioEssays 19: 257-262, 1997.

39. Gene Ontology C; Gene Ontology Consortium: Gene Ontology Consortium: Going forward. Nucleic Acids Res 43D: D1049-D1056, 2015

40. Krivanek J, Soldatov RA, Kastriti ME, Chontorotzea T, Herdina AN, Petersen J, Szarowska B, Landova M, Matejova VK, Holla LI, et al: Dental cell type atlas reveals stem and differentiated cell types in mouse and human teeth. Nat Commun 11: 4816,2020

41. Beertsen W, Holmbeck K, Niehof A, Bianco P, Chrysovergis K, Birkedal-Hansen $\mathrm{H}$ and Everts V: On the role of MT1-MMP, a matrix metalloproteinase essential to collagen remodeling, in murine molar eruption and root growth. Eur J Oral Sci 110: 445-451, 2002.

42. Zhao W, Dong Y, Wu C, Ma Y, Jin Y and Ji Y: miR-21 overexpression improves osteoporosis by targeting RECK. Mol Cell Biochem 405: 125-133, 2015

43. Hu Y, Smith CE, Richardson AS, Bartlett JD, Hu JC and Simmer JP: MMP20, KLK4, and MMP20/KLK4 double null mice define roles for matrix proteases during dental enamel formation. Mol Genet Genomic Med 4: 178-196, 2015

44. Dalmay T and Edwards DR: MicroRNAs and the hallmarks of cancer. Oncogene 25: 6170-6175, 2006.

45. Esquela-Kerscher A and Slack FJ: Oncomirs - microRNAs with a role in cancer. Nat Rev Cancer 6: 259-269, 2006.

46. Zhu Q, Wang Z, Hu Y, Li J, Li X, Zhou L and Huang Y: miR-21 promotes migration and invasion by the miR-21-PDCD4-AP-1 feedback loop in human hepatocellular carcinoma. Oncol Rep27: $1660-1668,2012$

47. GiovannettiE, FunelN, Peters GJ,Del Chiaro M,ErozenciLA, Vasile E, Leon LG, Pollina LE, Groen A, Falcone A, et al: MicroRNA-21 in pancreatic cancer: Correlation with clinical outcome and pharmacologic aspects underlying its role in the modulation of gemcitabine activity. Cancer Res 70: 4528-4538, 2010.

This work is licensed under a Creative Commons Attribution-NonCommercial-NoDerivatives 4.0 International (CC BY-NC-ND 4.0) License. 